Pacific Journal of Mathematics

ON THE ASYMPTOTIC BEHAVIOR OF LARGE PRIME 


\title{
ON THE ASYMPTOTIC BEHAVIOR OF LARGE PRIME FACTORS OF INTEGERS
}

\author{
K. Alladi AND P. ERDös
}

We prove results on the asymptotic behavior of large prime factors of the integers. The basic idea of the paper is that if $k$ is any fixed integer, then the $k$ th largest prime factor of $n$, denoted by $P_{k}(n)$ is generally much bigger than $\sum_{j>k} P_{j}(n)$. We give precise estimates of this phenomenon. This paper is a sequel to an earlier paper by the authors.

1. Notations and definitions. Throughout this paper the letters $p$ and $q$, with or without subscript will denote primes.

Let $n=\prod_{i=1}^{r} p_{i}^{\alpha_{i}}, p_{1}>p_{2}>\cdots>p_{r}$ be the canonical decomposition of an integer $n>1$ into primes. We set

$$
A(n)=\sum_{i=1}^{r} \alpha_{i} p_{i}, \quad A^{*}(n)=\sum_{i=1}^{r} p_{i}
$$

and

$$
\Omega(n)=\sum_{i=1}^{r} \alpha_{i}, \quad \omega(n)=r .
$$

Let $A(1)=A^{*}(1)=\Omega(1)=\omega(1)=0$.

We may define the $k$ th largest prime factor in two ways depending on whether we want to count prime factors according to multiplicity or not. To be more precise set

$$
\begin{aligned}
P_{k}^{*}(n) & =p_{k} \text { for } k \leqq \omega(n) \\
& =0 \text { for } k>\omega(n) .
\end{aligned}
$$

We may also define

$$
\begin{array}{ll}
P_{1}(n)=p_{1} \\
P_{k}(n)=P_{1}\left(\frac{n}{P_{1}(n) \cdot P_{2}(n) \cdots P_{k-1}(n)}\right), & 1<k \leqq \Omega(n) \\
P_{k}(n)=0 \text { for } k>\Omega(n) . &
\end{array}
$$

Observe that $P_{1}(n)=P_{1}^{*}(n)$.

The terms "average order" and "normal order" will mean the following: Let $f$ be an arithmetic function and set

$$
F(x)=\sum_{1 \leqq n \leqq x} f(n) .
$$

Suppose $g$ is a monotonic function such that 


$$
\lim _{x \rightarrow \infty} \frac{G(x)}{F(x)}=1
$$

where

$$
G(x)=\sum_{1 \leqq n \leqq x} g(n)
$$

then $f$ has average order $g$. Next, we say that two functions $f$ and $g$ are "nearly the same almost always" if for each $\varepsilon>0$

$$
\lim _{x \rightarrow \infty} \frac{\psi_{\varepsilon}(x)}{x}=1
$$

where

$$
\psi_{\varepsilon}(x)=\sum_{\substack{1 \leq n \leq x \\ 1-\epsilon<(f(n) \mid g(n))<1+\varepsilon}} 1
$$

If in (1.9) and (1.8), the function $g$ is monotonic, we say that $f$ has normal order $g$.

Consider the sum

$$
\psi(x, y)=\sum_{\substack{1 \leq n \leqq x \\ P_{1}(n) \leqq y}} 1
$$

If $\alpha \geqq 1$ is a real number and $y=x^{1 / \alpha}$, it is well known (see [10]) that

$$
\rho(\alpha)=\lim _{x \rightarrow \infty} \frac{\psi\left(x, x^{1 / \alpha}\right)}{x}
$$

exists. The limit in (1.11) is also defined if $-\infty<\alpha<1$ and

$$
\rho(\alpha)=\left\{\begin{array}{rr}
1 & 0 \leqq \alpha<1 \\
0 & -\infty<\alpha<0 .
\end{array}\right.
$$

The function $\rho(\alpha)$ is a monotonic decreasing continuous function of $\alpha$ for $\alpha \geqq 1$.

Finally we define the sums

$$
\begin{array}{ll}
S_{1}(x, k)=\sum_{2 \leqq n \leqq x} \frac{A(n)-P_{1}(n)-\cdots-P_{k-1}(n)}{P_{1}(n)}, & k \geqq 1 \\
S_{2}(x, k)=\sum_{2 \leqq n \leqq x} \frac{A^{*}(n)-P_{1}^{*}(n)-\cdots-P_{k-1}^{*}(n)}{P_{1}(n)}, & k \geqq 1 \\
S_{3}(x, k)=\sum_{2 \leqq n \leqq x} \frac{P_{k}(n)}{P_{1}(n)}, & k \geqq 1 \\
S_{4}(x, k)=\sum_{2 \leqq n \leqq x} \frac{P_{k}^{*}(n)}{P_{1}(n)}, & k \geqq 1 .
\end{array}
$$


The aim of this paper is to obtain estimates for these sums $S_{i}(x, k)$, $i=1,2,3,4$.

2. General background and main theorem. The results in this paper are in continuation of those in $\S 2$ of [2].

It is a well known theorem of Hardy and Ramanujan [6], [7] that the functions $\Omega(n)$ and $\omega(n)$ both have average and normal order $\log \log n=g(n)$. This means that a number $n$ usually has $\log \log n$ prime factors and most of them occur square free. Thus it is natural to expect the large prime factors to occur with multiplicity one, most of the time. So one should be able to show that the functions $A$ and $A^{*}$ have the same average order. In an earlier paper [2] we showed this to be true and much more.

Not only do $A$ and $A^{*}$ have the same average order, but the function $P_{1}(n)$ dominates the sums in (1.1) to such an extent that $A, A^{*}$ and $P_{1}$ have the same average order. More generally $A(n)-$ $P_{1}(n)-\cdots-P_{k-1}(n)$ and $P_{k}(n)$ have the same average order. It was observed in [1] that the functions $P_{k}^{*}(n)$ and $A^{*}(n)-P_{1}^{*}(n)-\cdots-$ $P_{k-1}^{*}(n)$ also have the same average order as $P_{k}(n)$, since the asymptotic analysis in [2] remains unaffected if the weak inequalities are replaced by strict ones. Thus we restate (without proof) the main theorem in [2] in a more complete form:

THEOREM A. If $k$ is a fixed positive integer then

$$
\begin{gathered}
\sum_{1 \leqq n \leqq x}\left\{A(n)-P_{1}(n)-\cdots-P_{k-1}(n)\right\} \sim \sum_{1 \leqq n \leqq x} P_{k}(n) \sim \sum_{1 \leqq n \leqq x} P_{k}^{*}(n) \\
\sim \sum_{1 \leqq n \leqq x}\left\{A^{*}(n)-P_{1}^{*}(n)-\cdots-P_{k-1}^{*}(n)\right\} \sim a_{k} \frac{x^{1+1 / k}}{(\log x)^{n}}
\end{gathered}
$$

where $a_{k}$ is a constant depending only on $k$, and is a rational multiple of $\zeta(1+1 / k)$ where $\zeta$ is the Riemann zeta function. In addition for each $k \geqq 1$

$$
\sum_{1 \leqq n \leqq x}\left\{A(n)-A^{*}(n)\right\}=x \log \log x+O(x)=o\left(\sum_{1 \leqq n \leqq x} P_{k}^{*}(n)\right) .
$$

Theorem A says that the average order in (2.1) is $g(n)=$ $a_{k}^{*} \cdot n^{1 / k} /(\log n)^{k}$ where $a_{k}^{*}=a_{k} \cdot(1+1 / k)$. An average is essentially influenced by two things-(i) the abnormally large values of a function, which certainly contribute to (2.1) and (ii) the values a function takes most often.

The question now arises whether $A, A^{*}$, and $P_{1}$ are nearly the same almost always. The main theorem stated below answers this question in the affirmative. 
TheOREM B. If $k$ is a fixed positive integer then

$$
S_{1}(x, k) \sim S_{2}(x, k) \sim S_{3}(x, k) \sim S_{4}(x, k) \sim a_{k}^{\prime} \frac{x}{(\log x)^{k-1}}
$$

where $a_{1}^{\prime}=1$ and $a_{k}^{\prime}$ for $k>1$ is a constant depending only on $k$, and is a rational multiple of $e^{r}$ where $\gamma$ is Euler's constant. In addition for each $k \geqq 1$

(2.4) $\sum_{2 \leqq n \leqq x} \frac{A(n)-A^{*}(n)}{P_{1}(n)}=O\left(\frac{x}{e^{c} \sqrt{\log x \log \log x}}\right)=o\left(\sum_{2 \leqq n \leqq x} \frac{P_{k}^{*}(n)}{P_{1}(n)}\right)$

where $c$ is an absolute constant $>0$.

3. Consequences and motivation. Statements (2.3) and (2.4) may be looked upon as analogues to (2.1) and (2.2). Theorem A said that $A, A^{*}$ and $P_{1}$ have the same average order, $\pi^{2} n / 6 \log n,\left(a_{1}=\right.$ $\pi^{2} / 12$, see [2]). We can deduce from Theorem $B$ the following.

Corollary. The functions $A, A^{*}$ and $P_{1}$ are all nearly the same almost always. Also all three functions fail to possess a normal order.

Proof. Consider two arithmetic functions $f, g$ satisfying $f(n) \geqq$ $g(n)>0$. Suppose that

$$
\sum_{1 \leqq n \leqq x} \frac{f(n)}{g(n)} \sim x
$$

We rewrite (3.1) as

$$
\sum_{1 \leqq n \leqq x}\left\{\frac{f(n)}{g(n)}-1\right\}=o(x) .
$$

Since $f / g \geqq 1$ we infer from (3.2) that

$$
\left|\frac{\psi_{\varepsilon}(x)}{x}-1\right|<\frac{o(x)}{\varepsilon \cdot x} \longrightarrow 0 \text { as } x \longrightarrow \infty
$$

for each $\varepsilon>0$, where $\psi_{\varepsilon}(x)$ is as in (1.8). So $f$ and $g$ are nearly the same almost always. (We can deduce (3.3) also if $f(n) \leqq g(n)$ for all $n$ ).

Setting $k=1$ in (2.3) we see that (3.1) is true with $f=A(n)$ and $g(n)=P_{1}(n)$. Therefore $A$ and $P_{1}$ are nearly the same almost always. Since $A \geqq A^{*} \geqq P_{1}$, the same is true for all three functions.

Now to show that these three functions do not have normal orders it suffices to show that one of them does not. It follows easily from a theorem of Elliott [5] on additive functions 


$$
f(n)=\sum_{p \mid n} f(p),
$$

that $A^{*}$ does not have a normal order. That proves the corollary.

REMARK. Since $A(n) \geqq \log n$, it follows from (2.2) that

$$
\begin{aligned}
\left|\sum_{2 \leqq n \leqq x}\left\{\frac{A^{*}(n)}{A(n)}-1\right\}\right| & \leqq \sum_{2 \leqq n \leqq x} \frac{A(n)-A^{*}(n)}{\log n} \\
& =0\left(\frac{x \log \log x}{\log x}\right) .
\end{aligned}
$$

From (3.2), (3.3) and (3.5) we can deduce that $A$ and $A^{*}$ are nearly the same almost always.

Let us look a little more closely at (2.3) which for $f=A$ or $A^{*}$ and $g=P_{1}$ is a more accurate form of (3.1). We may rewrite (2.3) as

$$
\begin{aligned}
\sum_{2 \leqq n \leqq x} \frac{A^{*}(n)}{P_{1}(n)}=\sum_{2 \leqq n \leqq x} 1 & +\sum_{2 \leqq n \leqq x} \frac{P_{2}^{*}(n)}{P_{1}(n)}+\cdots \\
& +\sum_{1 \leqq n \leqq x} \frac{P_{k}^{*}(n)}{P_{1}(n)}+\cdots
\end{aligned}
$$

where

$$
\sum_{2 \leqq n \leqq x} \frac{P_{k}^{*}(n)}{P_{1}(n)} \sim a_{k}^{\prime} x /(\log x)^{k} .
$$

We show in $\S 5$ that

$$
a_{k}^{\prime}=\int_{1}^{\infty} \rho(s-k) s^{l-2} d s
$$

where $\rho$ is defined in (1.11). We deduce from (3.8) in $\S 6$ that $\alpha_{k}^{\prime}$ is a rational multiple of $e^{r}$ for $k>1$. The integral representation is investigated in $\S 6$ and this leads to pretty connections with some related problems.

The next section is devoted to obtaining upper and lower bounds for $S_{i}(x, k), i=1,2,3,4$. This enables us to deduce the first four asymptotic relations in (2.3). It is only $\S 5$ that we prove (3.7) and (3.8). But the upper bound method in $\S 4$ is used in $\S 5$ to take care of the error terms arising out of (3.6) and (2.3). For the reader who does not want to go through the detailed proof, see [1], where some of the ideas of this paper and an earlier paper by the authors [2] are summarized.

We now move on to the proofs of our results.

4. Upper and lower bounds. In what follows, $c_{1}, c_{2}, c_{3}, \cdots$ 
denote absolute positive constants whose precise values will not be our concern. Also $\exp \{x\}=e^{x}$. We begin by proving

THEOREM 1. There exists for each positive integer $k$ a constant $b_{k}$ and a real number $x_{0}=x_{0}(k)$ such that if $x \geqq x_{0}$ then $S_{i}(x, k)>$ $b_{k} \cdot x /(\log x)^{k-1}$ for $i=1,2,3,4$.

To prove this we need

LEMMA 1. Let $s$ be a positive real number. Then

$$
\sum_{p>x} \frac{1}{p(\log p)^{s}}=\frac{1}{s(\log x)^{s}}+O\left(\exp \left\{-c_{1} \sqrt{\log x\}}\right) .\right.
$$

Proof. We use the Prime Number Theorem [4], [9] in the form

$$
|\pi(x)-l i(x)|=O\left(x \exp \left\{-c_{2} \sqrt{\log x}\right\}\right) .
$$

Now write

$$
\begin{aligned}
\sum_{p>x} \frac{1}{p(\log p)^{s}}= & \int_{x^{+}}^{\infty} \frac{d \pi(y)}{y(\log y)^{s}}=\int_{x}^{\infty} \frac{d y}{y(\log y)^{s+1}}+\int_{x^{+}}^{\infty} \frac{d\{\pi(y)-l i(y)\}}{y(\log y)^{s}} \\
= & \frac{1}{s(\log x)^{s}}+\left.\frac{\pi(y)-l i(y)}{y(\log y)^{s}}\right|_{x^{+}} ^{\infty} \\
& +O\left(\int_{x^{+}}^{\infty} \frac{\{\pi(y)-l i(y)\}}{y^{2}(\log y)^{s+1}} d y\right) .
\end{aligned}
$$

Lemma 1 follows from (4.1) and (4.2).

Proof of Theorem 1. It suffices to prove Theorem 1 for the smallest of the four sums $S_{4}(x, k)$.

Assume first that $k>1$. For $x$ sufficiently large choose a prime $p_{1}$ in the interval

$$
k ! x^{1 / k+1} \leqq p_{1} \leqq x^{1 / k}
$$

Now choose primes $p_{2}, p_{3}, \cdots, p_{k}$ satisfying

$$
\frac{p_{1}}{k}<p_{k}<\frac{p_{1}}{k-1}<p_{k-1}<\frac{p_{1}}{k-2}<\cdots<\frac{p_{1}}{3}<p_{3}<\frac{p_{1}}{2}<p_{2}<p_{1}
$$

Consider any multiple $m \leqq x$ of $p_{1} p_{2} \cdots p_{k}$

$$
m=n^{\prime} p_{1} p_{2} \cdots p_{k} \text {. }
$$

Because of (4.3) and (4.4) we have

$$
p_{1} p_{2} \cdots p_{k} \leqq x
$$


and

$$
n^{\prime} \leqq \frac{m}{p_{1} \cdots p_{k}} \leqq \frac{x}{(k !)^{n-1} x^{k / k+1}}<x^{1 / k+1}
$$

By (4.7) and (4.4)

$$
P_{1}\left(n^{\prime}\right) \leqq n^{\prime}<x^{1 / k+1} \leqq(k-1) ! x^{1 / k+1}<p_{k} .
$$

Thus by (4.5) and (4.8) we see that $P_{k}^{*}(m)=p_{k}$. So any multiple $\leqq x$ of $p_{1} \cdots p_{k}$ has $p_{k}$ as its $k$ th largest prime factor $\left(P_{k}^{*}\right)$. So

$$
S_{4}(x, k)=\sum_{2 \leqq n \leqq x} \frac{P_{k}^{*}(n)}{P_{1}(n)} \geqq \sum_{\substack{n \leq n \leq x \\ n=p_{1} \ldots p_{k} \\\left(p_{i}\right. \text { satisfying (4.4)) }}} \frac{P_{k}^{*}(n)}{P_{1}(n)} .
$$

We can estimate the second sum in (4.9) by using the well known result [11]

$$
\sum_{p \leqq k} \frac{1}{p}=\log \log x+c_{3}+O\left(\exp \left\{-c_{4} \sqrt{\log x}\right\}\right) .
$$

Observe that the second sum in (4.9) is

$$
\begin{aligned}
& \sum_{k ! x^{1 /(k+1)} \leqq p_{1} \leqq x^{1 / k}} \sum_{p_{1} / 2<p_{2}<p_{1}} \sum_{p_{1} / 3<p_{3}<p_{1} / 2} \cdots \sum_{p_{1} / k<p_{k}<p_{1} /(k-1)} \frac{x}{p_{1} \cdots p_{k}} \cdot \frac{p_{k}}{p_{1}} \\
& =x \sum_{k^{n} x^{1 /(k+1)} \leqq p_{1} \leqq x^{1 / k}} \frac{1}{p_{1}^{2}}\left\{\left(\prod_{j=2}^{k-1}\left(\sum_{p_{1} / j<p_{j}<p_{1} /(j-1)} \frac{1}{p_{j}}\right)\right) \cdot\left(\sum_{p_{1} / k<p_{k}<p_{1} /(k-1)} 1\right)\right\} \\
& \geqq b_{k}^{\prime} x \sum_{k^{\prime} x^{1 /(k+1)} \leqq p_{1} \leqq x^{1 / k}} \cdot \frac{1}{p_{1}\left(\log p_{1}\right)^{k-1}}>b_{k} x /(\log x)^{k-1}
\end{aligned}
$$

by virtue of Lemma 1 and (4.10). Theorem 1 follows from (4.9) and (4.11), for $k>1$. For $k=1$, Theorem 1 is trivially true.

Now for an upper bound.

Theorem 2. All four sums $S_{i}(x, k), i=1,2,3,4$ are $O\left(x /(\log x)^{k-1}\right)$ where $k$ is an integer $\geqq 1$, and the O-constant depends only on $k$.

We need a few preliminary results before proving Theorem 2 .

LEMma 2. Let $k$ be a nonnegative integer and

$$
S_{k}^{*}(x)=\sum_{1 \leqq p \leqq x}(\log \log x-\log \log p)^{k} .
$$

Then

$$
S_{k}^{*}(x)=\frac{k ! x}{(\log x)^{k+1}}+O_{k}\left(\frac{x \cdot \log \log x}{(\log x)^{k+2}}\right)
$$


Proof. If we write $S_{k}^{*}(x)$ as a Stieltjes integral, use the fact that

$$
d \pi(y)=\frac{d y}{\log y}+d\{\pi(y)-l i(y)\},
$$

integrate the second integral by parts and then use (4.1) we get

$$
S_{k}^{*}(x)=\int_{4}^{x} \frac{(\log \log x-\log \log y)^{k}}{\log y} d y+O\left(\frac{x}{(\log x)^{k+2}}\right) .
$$

Next

$$
\begin{aligned}
T_{k}(x)= & \int_{4}^{x} \frac{(\log \log x-\log \log y)^{k}}{\log y} d y \\
= & \left.(\log \log x-\log \log y)^{k} l i(y)\right|_{4} ^{x} \\
& \quad+k \int_{4}^{x} \frac{\operatorname{li}(y)(\log \log x-\log \log y)^{k-1}}{y \log y} d y \\
= & O\left((\log \log x)^{k}\right)+k \int_{4}^{x} \frac{\operatorname{li}(y)(\log \log x-\log \log y)^{k-1}}{y \log y} d y .
\end{aligned}
$$

But

$$
l i(y)=\frac{y}{\log y}+O\left(\frac{y}{\log ^{2} y}\right) .
$$

So the integral in (4.13) becomes

$$
\begin{aligned}
& k \int_{4}^{x} \frac{(\log \log x-\log \log y)^{k-1}}{\log ^{2} y}+O\left(\int_{4}^{x} \frac{(\log \log x-\log \log y)^{k-1}}{\log ^{3} y} d y\right) \\
& \quad=I_{1}+I_{2} .
\end{aligned}
$$

We split $I_{1}$ into

$$
I_{1}=k \int_{4}^{x /(\log x)^{k+3}}+k \int_{x /(\log x)^{k+3}}^{x} .
$$

Clearly in (4.16)

$$
\int_{4}^{x /(\log x)^{k+3}}=O\left(\frac{x(\log \log x)^{k-1}}{(\log x)^{k+3}}\right)=O\left(\frac{x}{(\log x)^{k+2}}\right) .
$$

Regarding the second integral in (4.16) we observe that

$$
\begin{aligned}
k \int_{x /(\log x)^{k+3}}^{x}=k\{ & \left.\frac{1}{\log x}+O\left(\frac{\log \log x}{\log ^{2} x}\right)\right\} \\
& \times \int_{x /(\log x)^{k+3}}^{x} \frac{(\log \log x-\log \log y)^{k-1}}{\log y} d y .
\end{aligned}
$$


Now the last integral in (4.18) is

$$
T_{k-1}(x)+O\left(\frac{x(\log \log )^{k-1}}{(\log x)^{k+3}}\right)=T_{k-1}(x)+O\left(\frac{x}{(\log x)^{k+2}}\right) .
$$

From the definition of $T_{k}$ we have

$$
T_{0}(x)=\frac{x}{\log x}+O\left(\frac{x}{\log ^{2} x}\right) .
$$

Now make the induction hypothesis that for $k \geqq 1$

$$
T_{k-1}(x)=\frac{(k-1) ! x}{(\log x)^{k}}+O\left(\frac{x(\log \log x)}{(\log x)^{k+1}}\right) .
$$

Then from equations (4.16) through (4.21) we deduce that

$$
I_{1}=\frac{k ! x}{(\log x)^{k+1}}+O\left(\frac{x \log \log x}{(\log x)^{k+2}}\right) .
$$

By analysis very similar to the above one can show that

$$
I_{2}=O\left(\frac{x}{(\log x)^{k+2}}\right) \text {. }
$$

So from (4.22), (4.23), (4.15) and (4.13) we see that (4.21) is true for $T_{k}(x)$ and so by induction for all $k \geqq 1$. Lemma 2 follows from (4.12) and (4.21). Then

LeMma 3. Let $x, y \geqq 4$ be real numbers and $k \geqq 0$ an integer.

$$
\begin{aligned}
\sum_{y \leqq p \leqq x} & \frac{(\log \log x-\log \log p)^{k}}{p}=\frac{(\log \log x-\log \log y)^{k+1}}{k+1} \\
& +O_{k}\left((\log \log x-\log \log y)^{k} \exp \left\{-c_{5} \sqrt{\log y}\right\}\right)
\end{aligned}
$$

Proof. As in the beginning of the proof of Lemma 2 we convert the above sum into a Stieltjes integral and replace $d \pi(y)$ by $d y / \log y$. Lemma 3 can be easily proved by making the substitution $\log \log x-$ $\log \log y=t$. We do not go through the details.

Proof of Theorem 2. It suffices to prove Theorem 2 for the largest of the four sums $S_{1}(x, k)$. That is we will show

$$
S_{1}(x, k)=\sum_{2 \leqq n \leqq x} \frac{A(n)-P_{1}(n)-\cdots-P_{k-1}(n)}{P_{1}(n)}=O\left(\frac{x}{(\log x)^{k-1}}\right)
$$

for $k \geqq 1$ an integer. We claim that it suffices to prove (4.24), for $k>1$ because for $k=1$ we have 


$$
\begin{aligned}
S_{1}(x, 1)=\sum_{2 \leqq n \leqq x} \frac{A(n)}{P_{1}(n)} & =\sum_{2 \leqq n \leqq x} 1+\sum_{2 \leqq n \leqq x} \frac{A(n)-P_{1}(n)}{P_{1}(n)} \\
& =x-1+S_{1}(x, 2) \\
& =O(x)
\end{aligned}
$$

assuming that (4.24) is true for $k=2$. So from now on we assume that $k>1$.

We write

$$
\begin{aligned}
\frac{A(n)-P_{1}(n)-P_{2}(n)-\cdots-P_{k-1}(n)}{P_{1}(n)}= & \frac{P_{k}(n)}{P_{1}(n)} \\
& +\frac{P_{k+1}(n)}{P_{1}(n)}+\cdots .
\end{aligned}
$$

Let us denote a general nonzero term of (4.27) by $p_{k} / p_{1}$. We would like to know how often this term occurs in $S_{1}(x, k)$. The term $p_{k} / p_{1}$ occurs as often as we can find integers $n=p_{1} p_{2} \cdots p_{k-1} \cdot p_{k} m \leqq x$ where the $p_{i}$ satisfy $p_{k} \leqq p_{k-1} \leqq \cdots \leqq p_{1}$ and $P_{1}(m) \leqq p_{k-1}$. If we fix the primes $p_{i}$ to satisfy these conditions then the number of such $n$ is given by

$$
\psi\left(\frac{x}{p_{1} p_{2} \cdots p_{k}}, p_{k-1}\right)
$$

where $\psi$ is defined in (1.10).

Thus we may rewrite (4.27) as

$$
\begin{aligned}
S_{1}(x, k)= & \sum_{2 \leqq p_{1} \leqq x} \sum_{p_{k} \leqq p_{1}} \sum_{p_{k} \leqq p_{k-1} \leqq p_{1}} \sum_{p_{k-1} \leqq p_{k-2} \leqq p_{1}} \cdots \\
& \cdots \sum_{p_{3} \leqq p_{2} \leqq p_{1}} \psi\left(\frac{x}{p_{1} \cdots p_{k}}, p_{k-1}\right) \frac{p_{k}}{p_{1}} .
\end{aligned}
$$

We first consider a subsum of (4.29) with a restriction on $p_{1}$. That is we choose $\beta$ with $0<\beta<1$, whose value will be specified later, and consider $p_{1}$ in (4.29) satisfying $x^{\beta} \leqq p_{1} \leqq x$. We shall get an upper bound for this sum.

Observe that the sum in (4.29) with this extra condition on $p_{1}$ is

$$
\begin{aligned}
& \leqq \sum_{x \beta \leqq p_{1} \leqq x} \sum_{p_{k} \leqq p_{1}} \sum_{p_{k} \leqq p_{k-1} \leqq p_{1}} \cdots \sum_{p_{3} \leqq p_{2} \leqq p_{1}} \frac{x}{p_{1} \cdots p_{k}} \cdot \frac{p_{k}}{p_{1}} \\
& =x \sum_{x \beta \leqq p_{1} \leqq x} \frac{1}{p_{1}^{2}} \sum_{p_{k} \leqq p_{1}} 1 \sum_{p_{k} \leqq p_{k-1} \leqq p_{1}} \frac{1}{p_{k-1}} \cdots \sum_{p_{3} \leqq p_{2} \leqq p_{1}} \frac{1}{p_{2}} .
\end{aligned}
$$

(Note: If $k=2$ in (4.30) we have only

$$
x \sum_{x \beta \leqq p_{1} \leqq x} \frac{1}{p_{1}^{2}} \sum_{p_{2} \leqq p_{1}} 1
$$


and no other terms. For $k>2$, there is no confusion in (4.30).) Because of this difference assume for the moment that $k>2$. Then if we use Lemma 3 we infer

$$
\sum_{p_{3} \leqq p_{2} \leqq p_{1}} \frac{1}{p_{2}}=O\left(\log \log p_{1}-\log \log p_{3}\right) .
$$

Again by Lemma 3 and (4.32)

$$
\sum_{p_{4} \leqq p_{3} \leqq p_{1}} \frac{1}{p_{3}} \sum_{p_{3} \leqq p_{2} \leqq p_{1}} \frac{1}{p_{2}}=O\left(\left(\log \log p_{1}-\log \log p_{4}\right)^{2}\right) .
$$

Iterating this process we get in (4.30) for $k>2$

$$
O\left(x \sum_{x^{\beta} \leqq p_{1} \leqq x} \frac{1}{p_{1}^{2}} \sum_{p_{k} \leqq p_{1}}\left(\log \log x-\log \log p_{k}\right)^{k-2}\right)
$$

by repeated use of Lemma 3. Now observe that because of (4.31) we see that (4.34) is true even for $k=2$. Thus for $k \geqq 2$, we may replace (4.30) by (4.34). Thus from now on we drop the assumption $k>2$, but of course still assume $k>1$.

To estimate (4.34) we use Lemma 2 which gives

$$
O\left(x \sum_{x \beta \leqq p_{1} \leqq x} \frac{1}{p_{1}\left(\log p_{1}\right)^{k-1}}\right) .
$$

Finally Lemma 1 and (4.35) imply that the sum in (4.35) and hence in $(4.30)$ is

$$
O\left(\frac{x}{\beta^{k-1}(\log x)^{k-1}}\right)
$$

where the constant on the $O$-term in (4.36) depends only on $k$ and not on $\beta$.

So (4.36) gives a bound for the sum in (4.29) with the condition $x^{\beta} \leqq p_{1} \leqq x$. For the sum corresponding to $p_{1} \leqq x^{\beta}$ we write

$$
\sum_{2 \leqq p_{1} \leqq x^{\beta}}=\sum_{m=0}^{\infty} \sum_{x^{\beta / 2} 2^{m+1} \leqq p_{1} \leqq x^{\beta / 2} m} \cdot
$$

To estimate (4.37) we use the following result of de Bruijn [3]; If $y=x^{1 / \alpha}$ then

$$
\psi(x, y)=O\left(x \exp \left\{-c_{6} \alpha\right\}\right) .
$$

In (4.37) consider the case

$$
x^{\beta / 2^{m+1}} \leqq p_{1} \leqq x^{\beta / 2^{m}} .
$$

Then in (4.29) with the restriction (4.39) on $p_{1}$ we have from (4.38) 
the following:

(4.40) $\quad \alpha=\frac{\log \left(x / p_{1} \cdots p_{k}\right)}{\log p_{k-1}} \geqq\left(1-\frac{k \beta}{2^{m}}\right) /\left(\frac{\beta}{2^{m}}\right)=\frac{2^{m}-k \beta}{\beta}$.

We choose $\beta=\beta(k)$, depending on $k$, so small that

$$
\alpha \geqq \frac{2^{m}-k \beta}{\beta}>\frac{2^{m-1}}{\beta} .
$$

Then by (4.38), (4.39), and (4.41) we will have in (4.29) for the subsum corresponding to $(4.39)$

$$
\psi\left(\frac{x}{p_{1} \cdots p_{k}}, p_{k-1}\right)=O\left(\frac{x}{p_{1} \cdots p_{k}} \exp \left\{-c_{6} 2^{m-1} / \beta\right\}\right) .
$$

If we substitute (4.42) in (4.29) and analyze this sum just the way we derived (4.36) we get

$$
\begin{aligned}
& O\left(\frac{x}{\left\{\log \left(x^{\beta / 2^{m+1}}\right)\right\}^{k-1}} \exp \left\{-c_{6} 2^{m-1} / \beta\right\}\right) \\
& \quad=O\left(\frac{x\left(2^{m+1} / \beta\right)^{k-1}}{(\log x)^{k-1} \exp \left\{c_{6} 2^{m-1} / \beta\right\}}\right) .
\end{aligned}
$$

But then

$$
\sum_{m=0}^{\infty} \frac{\left(2^{m+1} / \beta\right)^{k-1}}{\exp \left\{c_{6} 2^{m-1} / \beta\right\}}<\infty
$$

This means that (4.43), (4.42), and (4.36) imply that in (4.29)

$$
S_{1}(x, k)=O\left(x /(\log x)^{k-1}\right)
$$

for $k>1$. That completes the proof of Theorem 2 .

It is interesting to note that Theorems 1 and 2 actually imply the first four asymptotic relations in Theorem B, as will be shown below. Before establishing this we prove the last part of Theorem B namely

THEOREM 3. For each positive integer $k$ we have

$$
\begin{aligned}
\sum_{2 \leqq n \leqq x} \frac{A(n)-A^{*}(n)}{P_{1}(n)} & =O\left(x \exp \left\{-c_{7} \sqrt{\log x \log \log x}\right\}\right) \\
& =o\left(\sum_{2 \leqq n \leqq x} \frac{P_{k}^{*}(n)}{P_{1}(n)}\right) .
\end{aligned}
$$

Proof. First let $1 \leqq y \leqq x$ and $y=x^{1 / \alpha}$. N. G. de Bruijn [3] showed that if $3<\alpha<4 y^{1 / 2} / \log y$ then 
(4.44) $\psi(x, y)=O\left(x \log ^{2} y \exp \left\{-\alpha \log \alpha-\alpha \log \log \alpha+c_{8} \alpha\right\}\right)$.

Take $y=\exp \{\sqrt{\log x \log \log x}\}$. Then from (4.44) we have

$$
\psi(x, y)=O\left(x \exp \left\{-c_{9} \sqrt{\log x \log \log x\}}\right) .\right.
$$

Next observe that

$$
\frac{A(n)-A^{*}(n)}{P_{1}(n)} \leqq \Omega(n)=O(\log n) .
$$

We now split

$$
\sum_{2 \leqq n \leqq x} \frac{A(n)-A^{*}(n)}{P_{1}(n)}=\sum_{\substack{2 \leq n \leq x \\ P_{1}(n) \leq y}}+\sum_{\substack{2 \leq n \leq x \\ P_{1}(n)>y}}=\sum_{1}+\sum_{2} .
$$

Clearly from (4.46) and (4.45)

$$
\sum_{1}=O(\log x \cdot \psi(x, y))=O\left(x \exp \left\{-c_{10} \sqrt{\log x \log \log x}\right\}\right)
$$

But then by Theorem A, (2.2), we have

$$
\begin{aligned}
\sum_{2} & \leqq \exp \{-\sqrt{\log x \log \log x}\} \\
& =O\left(x \operatorname { e x p } \left\{-c_{11} \sqrt{\log x \log \log x\})} .\right.\right.
\end{aligned}
$$

The first equation in Theorem 3 follows from (4.47), (4.48) and (4.49). The second equation is a consequence of Theorem 1 . That proves Theorem 3.

THEOREM 4. For every integer $k \geqq 1$ we have

$$
S_{1}(x, k) \sim S_{2}(x, k) \sim S_{3}(x, k) \sim S_{4}(x, k) .
$$

Proof. The smallest of the four sums is $S_{4}(x, k)$. By Theorem 1

$$
S_{1}(x, k) \geqq S_{4}(x, k)>b_{k} x /(\log x)^{k-1} .
$$

The largest of the four sums is $S_{1}(x, k)$. Consider the difference

$$
\begin{aligned}
S_{1}(x, k)-S_{4}(x, k)= & \sum_{2 \leqq n \leqq x} \frac{A(n)-P_{1}(n)-\cdots-P_{k-1}(n)-P_{k}^{*}(n)}{P_{1}(n)} \\
= & \sum_{2 \leqq n \leqq x} \frac{A(n)-P_{1}(n)-\cdots-P_{k}(n)}{P_{1}(n)} \\
& \quad+\sum_{2 \leqq n \leqq x} \frac{P_{k}(n)-P_{k}^{*}(n)}{P_{1}(n)} \\
= & S_{1}(x, k+1)+\sum_{2 \leqq n \leqq x} \frac{P_{k}(n)-P_{k}^{*}(n)}{P_{1}(n)} .
\end{aligned}
$$

By Theorem 2 


$$
S_{1}(x, k+1)=O\left(x /(\log x)^{k}\right)
$$

But then

$$
\text { (4.53) } \begin{aligned}
A(n)-A^{*}(n)=\sum_{j=1}^{\infty} P_{j}(n)-\sum_{j=1}^{\infty} P_{j}^{*}(n) & =\sum_{j=1}^{\infty}\left\{P_{j}(n)-P_{j}^{*}(n)\right\} \\
& \geqq P_{k}(n)-P_{k}^{*}(n) .
\end{aligned}
$$

So by (4.53) and Theorem 3 we have

$$
\begin{aligned}
\sum_{2 \leqq n \leqq x} \frac{P_{k}(n)-P_{k}^{*}(n)}{P_{1}(n)} & \leqq \sum_{2 \leqq n \leqq x} \frac{A(n)-A^{*}(n)}{P_{1}(n)} \\
& =O\left(x \exp \left\{-c_{7} \sqrt{\log x \log \log x}\right\}\right) .
\end{aligned}
$$

Clearly from (4.51), (4.52) and (4.54)

$$
S_{1}(x, k)-S_{4}(x, k)=O\left(x /(\log x)^{k}\right) .
$$

Thus from (4.55) and (4.50) we deduce

$$
S_{1}(x, k) \sim S_{4}(x, k) .
$$

But since these are the smallest and largest sums, Theorem 4 follows from (4.56).

While proving Theorem 2 we did not use Lemmas 1, 2, and 3 in the forms in which they were stated, but used only the upper bounds they implied. These lemmas will play a role in obtaining asymptotic estimates, which we take up in the next section. We refer to the method of proof of Theorem 2 (namely the choice of $\beta$ and the convergence of the series (4.43)), as the "upper bound method" and use this method to take care of the error terms arising out of the asymptotic estimates in what follows.

5. Asymptotic estimates. Our goal in this section is to prove

THEOREM 5. Let $k$ be a positive integer. Then all the four sums $S_{i}(x, k), i=1,2,3,4$ are asymptotically equal to

$$
a_{k}^{\prime} x /(\log x)^{k-1}
$$

where

$$
a_{k}^{\prime}=\int_{1}^{\infty} \rho(s-k) s^{k-2} d s
$$

We need some lemmas before we go to the proof.

LeMma 4. If $\alpha \geqq 1$ and $\varepsilon>0$ then 


$$
|\rho(\alpha+\varepsilon)-\rho(\alpha)|=O\left(\frac{\varepsilon}{\Gamma(\alpha+1)}\right)
$$

Proof. It is well known (see [10]) that $\rho$ satisfies

$$
\rho(\alpha)=1-\int_{1}^{\alpha} \frac{\rho(t-1) d t}{t} .
$$

Furthermore (see [10], [3])

$$
\rho(\alpha)<\frac{c_{12}}{\Gamma(\alpha+1)} .
$$

Combining (5.1) and (5.2) we get

$$
0 \leqq \rho(\alpha)-\rho(\alpha+\varepsilon)=\int_{\alpha}^{\alpha+\varepsilon} \frac{\rho(t-1)}{t} d t \leqq \frac{\rho(\alpha-1) \varepsilon}{\alpha}=O\left(\frac{\varepsilon}{\Gamma(\alpha+1)}\right)
$$

because $\rho$ is monotonic decreasing.

LEMMA 5. There exists constants $c_{13}, c_{14}$ and $c_{15}$ such that if $\alpha \geqq 1$ and $y=x^{1 / \alpha}, x \geqq 1$, then

$$
\left|\psi\left(x, x^{1 / \alpha}\right)-x \rho(\alpha)\right| \leqq 2 \max \left(\frac{c_{13} x \alpha^{2}}{\exp \left\{c_{14} \sqrt{\log y\}}\right.}, \frac{c_{15} x \alpha}{e^{\alpha / 4} \cdot \log x}\right) .
$$

Proof. Lemma 5 is obtained by combining certain results of de Bruijn [3]. For the function $\Lambda\left(x, x^{1 / \alpha}\right)$ defined by de Bruijn, it is known

$$
\left|\psi\left(x, x^{1 / \alpha}\right)-\Lambda\left(x, x^{1 / \alpha}\right)\right|<c_{13} x \alpha^{2} \exp \left\{-c_{14} \sqrt{\log y}\right\}
$$

and

$$
\left|\Lambda\left(x, x^{1 / \alpha}\right)-x \rho(\alpha)\right|<c_{15} x \alpha /\left(e^{\alpha / 4} \cdot \log x\right) .
$$

Lemma 5 follows from (5.3) and (5.4).

Proof of Theorem 5. Because of Theorem 4 it suffices to prove Theorem 5 for one of sums $S_{i}(x, k)$. We consider $S_{1}(x, k)$. So we start with (4.29). (We assume $k>1$ since Theorem 5 is trivially true for $k=1$. (See (5.1), (5.2) and Theorem 4.)

In (4.29) we first look at the contribution due to numbers for which

$$
\frac{p_{k}}{p_{1}}<\frac{1}{\left(\log p_{1}\right)^{k+1}} .
$$

We will get an upper bound for the contribution due to such numbers. 
Let $0<\beta<1$ be a real number whose value will be specified later. Then write

$$
\sum_{2 \leqq p_{1} \leqq x}=\sum_{x^{\beta} \leqq p_{1} \leqq x}+\sum_{m=0}^{\infty} \sum_{x^{\beta / 2} m+1 \leqq p_{1} \leqq x^{\beta} 2^{m}} .
$$

In the interval $x^{\beta / 2^{m+1}} \leqq p_{1}<x^{\beta / 2^{m}}$ one has an upper bound for $\psi$ given in (4.42), while for $x^{\beta} \leqq p_{1} \leqq x$ we use the trivial upper bound

$$
\psi\left(\frac{x}{p_{1} \cdots p_{k}}, p_{k-1}\right) \leqq \frac{x}{p_{1} \cdots p_{k}} .
$$

Then for numbers satisfying (5.5) together with $x^{\beta} \leqq p_{1} \leqq x$, we have the following bound in (4.29)

$$
O\left(x \sum_{x \beta<p_{1} \leqq x} \frac{1}{p_{1}\left(\log p_{1}\right)^{k+1}} \sum_{p_{k} \leqq p_{1}} \frac{1}{p_{k}} \sum_{p_{k} \leqq p_{k-1} \leqq p_{1}} \frac{1}{p_{k-1}} \cdots \sum_{p_{3} \leqq p_{2} \leqq p_{1}} \frac{1}{p_{2}}\right) .
$$

Analysis similar to (4.32), (4.33) and (4.34) yields

$$
\begin{gathered}
O\left(x \sum_{x^{\beta} \leqq p_{1} \leqq x} \frac{1}{p_{1}\left(\log p_{1}\right)^{k+1}} \sum_{p_{k} \leqq p_{1}} \frac{1}{p_{k}}\left(\log \log p_{1}-\log \log p_{k}\right)^{k-2}\right) \\
\quad=O\left(x \sum_{x \beta \leqq p_{1} \leqq x} \frac{\left(\log \log p_{1}\right)^{k-1}}{p_{1}\left(\log p_{1}\right)^{k+1}}\right)=O\left(\frac{x(\log \log x)^{k-1}}{\beta^{k+1}(\log x)^{k+1}}\right)
\end{gathered}
$$

using Lemma 1 . To estimate the contribution due to integers satisfying (5.5) for the case $p_{1} \leqq x^{\beta}$, we use the decomposition of the last sum of (5.6). Then the upper bound method yields

$$
O\left(\frac{x(\log \log x)^{k-1}}{(\log x)^{k+1}}\right)=O\left(\frac{x}{(\log x)^{k}}\right)
$$

provided $\beta$ is suitably chosen. Thus from (5.9) and (5.10) we conclude that the contribution due to terms satisfying (5.5) is given by (5.10), and is smaller than the asymptotic term we are seeking.

Next we observe that the contribution due to terms for which $p_{1}=p_{1}(n)$ is small is negligible. For that purpose set

$$
y=\left(\exp \left\{(\log x)^{2 / 3}\right\}\right) \text {. }
$$

With $y$ as in (5.11) we have by (4.38)

$$
\psi(x, y)=O\left(x \exp \left\{-c_{16}(\log x)^{1 / 3}\right\}\right) .
$$

So, if $p_{1}=P_{1}(n) \leqq y$, then

$$
\begin{aligned}
& \sum_{2 \leqq n \leqq x} \frac{A(n)-P_{1}(n)-\cdots-P_{k-1}(n)}{P_{1}(n)} \leqq \sum_{\substack{2 \leq n \leq x \\
P_{1}(n) \leqq y}} \Omega(n) \leqq O\left(\sum_{\substack{2 \leq n \leq x \\
P_{1}(n) \leqq y}} \log x\right) \\
& \quad=O(\log x \psi(x, y))=O\left(x \exp \left\{-c_{17}(\log x)^{1 / 3}\right\}\right) .
\end{aligned}
$$


Because of (5.13) and (5.10), we assume from now on that

$$
\frac{p_{1}}{\left(\log p_{1}\right)^{k+1}} \leqq p_{k} \leqq p_{1} ; P_{1}(n)=p_{1}>\exp \left\{(\log x)^{2 / 3}\right\} .
$$

Once we assume (5.14) we can rewrite Lemma 5 as

$$
\begin{aligned}
& \psi\left(\frac{x}{p_{1} p_{2} \cdots p_{k}}, p_{k-1}\right)=x \rho\left(\frac{\log \left(x / p_{1} \cdots p_{k}\right)}{\log p_{k-1}}\right) \\
& \quad+O\left(\frac{x e^{-\alpha / 4} \cdot \alpha}{p_{1} \cdots p_{k} \log p_{k-1}}\right)
\end{aligned}
$$

where $\alpha=\log \left(x / p_{1} \cdots p_{k}\right) / \log p_{k-1}$.

The idea is to substitute (5.15) in (4.29). It is then easy to take care of the contribution due to the error term in (5.15) in (4.29) by observing that (5.14)

$$
\log p_{k-1} \geqq \log p_{k} \sim \log p_{1}>\frac{1}{2} \log p_{1}, \quad x \geqq x_{0} .
$$

This means if we substitute the $O$-term of (5.15) in (4.29), and use the upper bound method we get

$$
O\left(x /(\log x)^{k}\right) \text {. }
$$

The convergence of a series like (4.43) is ensured this time by the $e^{-\alpha / 4}$ term in (5.15). Since (5.17) is smaller than the asymptotic term we are seeking, we may forget the contribution of the $O$-term in (5.15), in the sum (4.29).

As to the leading term of (5.15) we observe that

$$
\rho\left(\frac{\log \left(x / p_{1} \cdots p_{k}\right)}{\log p_{k-1}}\right)=\rho\left(\frac{\log x-\sum_{i=1}^{k} \log p_{i}}{\log p_{k-1}}\right) .
$$

By (5.14) we have

$$
\log p_{i}=\log p_{1}+O\left(\log \log p_{1}\right), \quad 1 \leqq i \leqq k .
$$

Substituting (5.19) in (5.18) we get

$$
\rho\left(\frac{\log \left(x / p_{1} \cdots p_{k}\right)}{\log p_{k-1}}\right)=\rho\left\{\frac{\log x}{\log p_{1}}-k+O\left(\frac{\log x \cdot \log \log p_{1}}{\log ^{2} p_{1}}\right)\right\}
$$

Using Lemma 4 to estimate (5.20) we get

$$
\rho\left(\frac{\log x}{\log p_{1}}-k\right)+O\left(\frac{\log x \cdot \log \log p_{1}}{\log ^{2} p_{1} \cdot \Gamma(\alpha)}\right)
$$

where $\alpha$ is as in (5.15). 
Thus the factor $\rho$ in the leading term of (5.15) is equal to the quantity in (5.21). Recall that our idea is to substitute (5.15) in (4.29) and estimate the sum. The contribution of the $O$-terms in (5.21) can be obtained by the upper bound method. There is a log $x$ in the numerator, but a $\log ^{2} p_{1}$ in the denominator. This time the presence of $\Gamma(\alpha)$ in the denominator ensures convergence in a series like (4.43). Thus the upper bound method yields

$$
O\left(x \log \log x /(\log x)^{k}\right)
$$

as the contribution due to the $O$-term of (5.21). Thus we deduce that the main contribution from (4.29) comes by assuming (5.14) and replacing $\psi\left(x / p_{1} \cdots p_{k}, p_{k-1}\right)$ by

$$
\frac{x}{p_{1} \cdots p_{k}} \rho\left(\frac{\log x}{\log p_{1}}-k\right) \text {. }
$$

So we replace (4.29) by

$$
\begin{aligned}
& x \sum_{\exp \left\{(\log x)^{2 / 3}\right\} \leqq p_{1} \leqq x} \frac{\rho\left(\left(\log x / \log p_{1}\right)-k\right)}{p_{1}^{2}} \\
& \quad \times \sum_{p_{1} /\left(\log p_{1}\right) k+1 \leqq p_{k} \leqq p_{1}} \sum_{p_{k} \leqq p_{k-1} \leqq p_{1}} \frac{1}{p_{k-1}} \cdots \sum_{p_{3} \leqq p_{2} \leqq p_{1}} \frac{1}{p_{2}} .
\end{aligned}
$$

To estimate (5.24) we use Lemma 3. First we get

(5.25) $\sum_{p_{3} \leqq p_{2} \leqq p_{1}} \frac{1}{p_{2}}=\left(\log \log p_{1}-\log \log p_{3}\right)+O\left(\exp \left\{-c_{5} \sqrt{\log p_{3}}\right\}\right)$

The contribution due to the $O$-term in (5.25) in (5.24) is taken care of by the upper bound method. This time the presence of $\rho$ in (5.24) ensures convergence of a series like (4.43), because of (5.2). Actually every error term that arises in (5.24) by repeated use of Lemma 3 can be estimated by the upper bound method, yielding

$$
O\left(x \exp \left\{-c_{18} \sqrt{\log x}\right\}\right) .
$$

So we need only look at the leading terms arising out of Lemma 3 in (5.24). After $k$-2 applications of the lemma we are left with

$$
\begin{aligned}
& x \quad \sum_{\exp \left\{(\log x)^{2 / 3\}} \leqq p_{1} \leqq x\right.} \frac{\rho\left((\log x) /\left(\log p_{1}\right)-k\right)}{p_{1}^{2}} \\
& \quad \times \sum_{p_{1} /\left(\log p_{1}\right) k+1 \leqq p_{k} \leqq p_{1}} \frac{\left(\log \log p_{1}-\log \log p_{k}\right)^{k-2}}{(k-2) !} .
\end{aligned}
$$

In (5.27) we use Lemma 2 to get 


$$
\begin{aligned}
x & \sum_{\exp \left\{(\log x)^{2 / 3} \leq p_{1} \leq x\right.} \frac{\left.\rho\left((\log x) /\left(\log p_{1}\right)\right)-k\right)}{p_{1}^{2}} \\
\times & {\left[\frac{p_{1}}{\left(\log p_{1}\right)^{k-1}}+O\left(\frac{p_{1}\left(\log \log p_{1}\right)}{\left(\log p_{1}\right)^{k}}\right)\right] . }
\end{aligned}
$$

As before, the $O$-term in (5.28) contributes

$$
O\left(x \log \log x /(\log x)^{k}\right)
$$

by use of the upper bound method. Finally the leading term in (5.28) is estimated by writing it as a Stieltjes integral. That is

$$
\begin{aligned}
x & \sum_{\exp \left\{( \operatorname { l o g } x ^ { 2 / 3 } \} \left\{p_{1} \leq x\right.\right.} \frac{\rho\left((\log x) /\left(\log p_{1}\right)-k\right)}{p_{1}\left(\log p_{1}\right)^{k-1}} \\
= & x \int_{\operatorname{ep~}\left\{\left\{(\log x)^{2 / 3}\right\}^{-}\right.}^{x^{+}} \frac{\rho((\log x) /(\log y)-k) d \pi(y)}{y(\log y)^{k-1}} \\
= & x \int_{\exp \left\{(\log x)^{2 / 3\}}\right.}^{x} \frac{\rho((\log x) /(\log y)-k)}{y(\log y)^{k}} d y \\
& +x \int_{\exp \left\{(\log x)^{2 / 3}\right\}^{-}}^{x^{+}} \frac{\rho((\log x) /(\log y)-k)}{y(\log y)^{k-1}} \\
& \times d\{\pi(y)-l i(y)\}=I_{3}+I_{4} .
\end{aligned}
$$

We can bound $I_{4}$ rather easily. First observe that $|\rho| \leqq 1$. Ignoring $o$, we integrate by parts, and use (4.1) to deduce

$$
I_{4}=O\left(\left(x \exp \left\{-c_{19}(\log x)^{1 / 3}\right\}\right) .\right.
$$

To estimate $I_{3}$ write $y=x^{1 / s}$. Then

$$
\begin{aligned}
I_{3} & =\frac{x}{(\log x)^{k-1}} \int_{1}^{(\log x)^{1 / 3}} \rho(s-k) s^{k-2} d s \\
& =\frac{x}{(\log x)^{k-1}}\left[\int_{1}^{\infty}-\int_{(\log x)^{13}}^{\infty}\right] \\
& =a_{k}^{\prime} \frac{x}{(\log x)^{k-1}}+O\left(\frac{x}{(\log x)^{k}}\right)
\end{aligned}
$$

because of (5.2). So Theorem 5 follows from (5.32) and the preceding estimates.

REMARKs. Note that we have actually shown that

$$
S_{1}(x, k)=a_{k}^{\prime} \frac{x}{(\log x)^{k-1}}+O\left(\frac{x \log \log x}{(\log x)^{k}}\right) .
$$

Observe that $S_{1}(x, k)$ is the largest of the four sums and $S_{4}(x, k)$ is the smallest. Therefore, because of (4.55), we deduce a stronger form of Theorem 5, namely 


$$
S_{i}(x, k)=a_{k}^{\prime} \frac{x}{(\log x)^{k-1}}+O\left(\frac{x \log \log x}{(\log x)^{k}}\right)
$$

for $i=1,2,3,4$.

Thus we have proved all the statements of Theorem $B$, except the relation between $a_{k}$ and $e^{r}$. We do this in the next section.

6. The constants $a_{k}^{\prime}$. It is obvious from Theorem 4 or (5.1) and (5.2) that $\alpha_{1}^{\prime}=1$. So we suppose $k \geqq 2$. For $k \geqq 2$ write

$$
\begin{aligned}
a_{k}^{\prime} & =\int_{1}^{\infty} \rho(s-k) s^{k-2} d s=\int_{k}^{\infty} \rho(s-k) s^{k-2} d s=\int_{0}^{\infty} \rho(t)(t+k)^{k-2} d t \\
& =\sum_{j=0}^{k-2}\left(\begin{array}{c}
k-2 \\
j
\end{array}\right) k^{k-2-j} \int_{0}^{\infty} \rho(t) t^{j} d t=\sum_{j=0}^{k-2}\left(\begin{array}{c}
k-2 \\
j
\end{array}\right) k^{k-2-j} f_{j}
\end{aligned}
$$

where

$$
f_{j}=\int_{0}^{\infty} \rho(t) t^{j} d t
$$

In a recent paper, Knuth and Pardo [8], have studied the behavior of

$$
\psi_{k}(x, y)=\sum_{\substack{1 \leq n \leq x \\ P_{k}^{*}(x) \leqq y}} 1
$$

In the course of their investigations they show

$$
f_{j}=e^{r} g_{j}
$$

where $\gamma$ is Euler's constant and the $g_{j}$ are recursively defined by

$$
g_{0}=g_{1}=1, \quad g_{j}=\frac{1}{j} \sum_{1 \leqq i \leqq j}\left(\begin{array}{l}
j \\
i
\end{array}\right) g_{j-i}, \quad j \geqq 0 .
$$

Combining (6.2), (6.5), and (6.6) we infer that $a_{k}^{\prime}$ is a rational multiple of $e^{r}$ for $k \geqq 2$. For instance

$$
a_{2}^{\prime}=f_{0}=e^{r} g_{0}=e^{r} \text {. }
$$

That completes the proof of Theorem B.

Acknowledgments. K. Alladi is grateful to Professors R. Miech and E. G. Straus for their advice and encouragement. They went through an early draft of this paper and made helpful suggestions. We also thank the referee for his critical comments and suggestions.

\section{REFERENCES}

1. K. Alladi, Analogues to the Hardy-Ramanujan theorems, in Proceedings of the 
Conference of Number Theory and Computational Methods, Publications of "Matscience", Madras, India (1977).

2. K. Alladi and P. Erdös, On an additive arithmetic function, Pacific J. Math., 71 (2), (1977), 275-294.

3. N. G. de Bruijn, On the number of positive integers $\leqq \times$ and free of prime factors $>$ y, Indag. Math., 13 (1951), 50-60.

4. K. Chandrasekharan, Arithmetical Functions, Springer-Verlag, New York, 1970.

5. P.D. T. A. Elliott, On a problem of Hardy and Ramanujan, Mathematika 23, (1976), 10-17.

6. G. H. Hardy and S. Ramanujan, The normal number of prime factors of a number n, Quarterly J. Math., 58 (1917), 76-92.

7. G. H. Hardy and E. M. Wright, An Introduction to the Theory of Numbers, Oxford, Clarendon, 1960, 4-Ed.

8. D. E. Knuth and L. T. Pardo, Analysis of a simple factorisation algorithm, (to appear).

9. W. J. LeVeque, Topics in Number Theory, Vol. 2, Addison-Wesley, Reading, Mass., 1956.

10. K. Norton, Numbers with small prime factors and the least kth-power non-residue, Memoirs of the A. M. S., 106, Providence, Rhode Island, 1971.

11. J. B. Rosser and L. Schoenfeld, Approximate formulas for some functions of prime numbers, Illinois J. Math., 6 (1962), 64-94.

Received April 3, 1978 and in revised form October 16, 1978. This work was supported in part under National Science Foundation Grants No. MCS 77-01780 and MCS 78-02685.

University of CALIFoRnia

Department of Mathematics

Los ANgeles, California 90024

AND

Mathematics Institute

The Hungarian Academy of Sciences

BUdAPEST, HUNGARY

Current Address of first author: University of Michigan Department of Mathematics Ann Arbor, MI 48109 



\section{PACIFIC JOURNAL OF MATHEMATICS}

\section{EDITORS}

Donald BABBITT (Managing Editor)

University of California

Los Angeles, CA 90024

HUGo RossI

University of Utah

Salt Lake City, UT 84112

C. C. MOORE and ANDREW OGG

University of California

Berkeley, CA 94720

\section{J. DuGUNDJI}

Department of Mathematics

University of Southern California

Los Angeles, CA 90007

R. FINN and J. Milgram

Stanford University

Stanford, CA 94305

ASSOCIATE EDITORS
E. F. BECKENBACH
B. H. NeumanN
F. WOLF
K. YoSHIDA

\section{SUPPORTING INSTITUTIONS}

\author{
UNIVERSITY OF BRITISH COLUMBIA \\ CALIFORNIA INSTITUTE OF TECHNOLOGY \\ UNIVERSITY OF CALIFORNIA \\ MONTANA STATE UNIVERSITY \\ UNIVERSITY OF NEVADA, RENO \\ NEW MEXICO STATE UNIVERSITY \\ OREGON STATE UNIVERSITY \\ UNIVERSITY OF OREGON
}

\author{
UNIVERSITY OF SOUTHERN CALIFORNIA \\ STANFORD UNIVERSITY \\ UNIVERSITY OF HAWAII \\ UNIVERSITY OF TOKYO \\ UNIVERSITY OF UTAH \\ WASHINGTON STATE UNIVERSITY \\ UNIVERSITY OF WASHINGTON
}

The Supporting Institutions listed above contribute to the cost of publication of this Journal, but they are not owners or publishers and have no responsibility for its content or policies.

Mathematical papers intended for publication in the Pacific Journal of Mathematics should be in typed form or offset-reproduced, (not dittoed), double spaced with large margins. Please do not use built up fractions in the text of the manuscript. However, you may use them in the displayed equations. Underline Greek letters in red, German in green, and script in blue. The first paragraph or two must be capable of being used separately as a synopsis of the entire paper. Please propose a heading for the odd numbered pages of less than 35 characters. Manuscripts, in triplicate, may be sent to any one of the editors. Please classify according to the scheme of Math. Reviews, Index to Vol. 39. Supply name and address of author to whom proofs should be sent. All other communications should be addressed to the managing editor, or Elaine Barth, University of California, Los Angeles, California, 90024.

50 reprints to each author are provided free for each article, only if page charges have been substantially paid. Additional copies may be obtained at cost in multiples of 50 .

The Pacific Journal of Mathematics is issued monthly as of January 1966. Regular subscription rate: $\$ 84.00$ a year (6 Vols., 12 issues). Special rate: $\$ 42.00$ a year to individual members of supporting institutions.

Subscriptions, orders for numbers issued in the last three calendar years, and changes of address should be sent to Pacific Journal of Mathematics, P.O. Box 969, Carmel Valley, CA 93924, U.S.A. Older back numbers obtainable from Kraus Periodicals Co., Route 100, Millwood, NY 10546.

PUBLISHED BY PACIFIC JOURNAL OF MATHEMATICS, A NON-PROFIT CORPORATION

Printed at Kokusai Bunken Insatsusha (International Academic Printing Co., Ltd.). 8-8, 3-chome, Takadanobaba, Shinjuku-ku, Tokyo 160, Japan.

Copyright (C) 1979 by Pacific Journal of Mathematics Manufactured and first issued in Japan 


\section{Pacific Journal of Mathematics}

\section{Vol. 82 , No. 2 \\ February, 1979}

Krishnaswami Alladi and Paul Erdős, On the asymptotic behavior of large prime

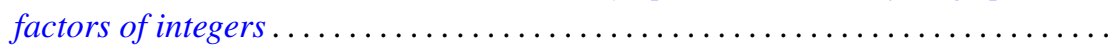

Alfred David Andrew, A remark on generalized Haar systems in $L_{p}$,

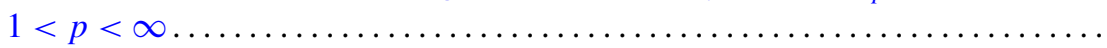

John M. Baker, A note on compact operators which attain their norm . . ........

Jonathan Borwein, Weak local supportability and applications to

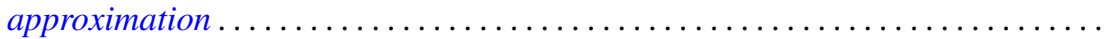

Tae Ho Choe and Young Soo Park, Wallman's type order compactification ........

Susanne Dierolf and Ulrich Schwanengel, Examples of locally compact

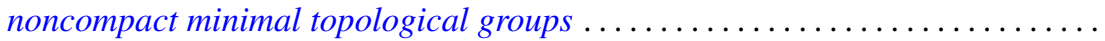

Michael Freedman, A converse to (Milnor-Kervaire theorem) $\times R$ etc. . . . . . . .

George Golightly, Graph-dense linear transformations ..................

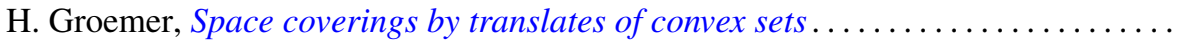

Rolf Wim Henrichs, Weak Frobenius reciprocity and compactness conditions in

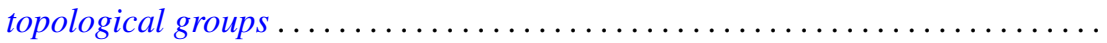

Horst Herrlich and George Edison Strecker, Semi-universal maps and universal

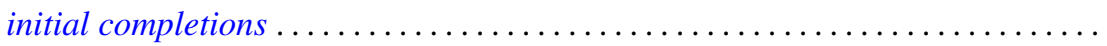

Sigmund Nyrop Hudson, On the topology and geometry of arcwise connected,

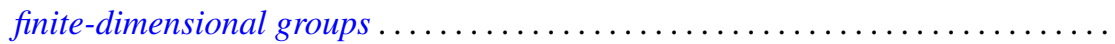

K. John and Václav E. Zizler, On extension of rotund norms. II .............

Russell Allan Johnson, Existence of a strong lifting commuting group of transformations. II.

Bjarni Jónsson and Ivan Rival, Lattice varieties covering the smallest nonmodular variety

Grigori Abramovich Kolesnik, On the order of Dirichlet L-functions .

Robert Allen Liebler and Jay Edward Yellen, In search of nonsolvable groups of

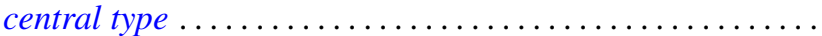

Wilfrido Martínez T. and Adalberto Garcia-Maynez Cervantes, Unicoherent plane Peano sets are $\sigma$-unicoherent ...

M. A. McKiernan, General Pexider equations. I. Existence of injective

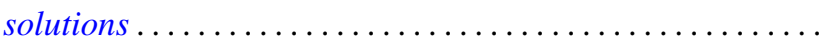

M. A. McKiernan, General Pexider equations. II. An application of the theory of webs.

Jan K. Pachl, Measures as functionals on uniformly continuous functions . .

Lee Albert Rubel, Convolution cut-down in some radical convolution algebras ...

Peter John Slater and William Yslas Vélez, Permutations of the positive integers

with restrictions on the sequence of differences. II . . .

Raymond Earl Smithson, A common fixed point theorem for nested spaces ....

Indulata Sukla, Generalization of a theorem of McFadden .... . . .

Jun-ichi Tanaka, A certain class of total variation measures of analytic measures.

Kalathoor Varadarajan, Modules with supplements .............. 